\title{
A PHENOMENOLOGICAL CONTENT ANALYSIS OF PSYCHOLOGICAL FLEXIBILITY IN UNIVERSITY STUDENTS OF LAHORE
}

\author{
Nazish Idrees Chaudhary*, Muhammad Shoaib Zafar ${ }^{\dagger}$ \\ \& Muhammad Rafiq Dar*
}

\begin{abstract}
Psychological flexibility is an emerging phenomenon worldwide. It has been shown that an adequate level of psychological flexibility supports with adjustment of an individual in a present situation. In European cultures, psychological flexibility has been associated with successful outcomes and adaptive behaviours for stress management. The phenomenology of a variable differs in different populations; the scarcity of an indigenous psychological flexibility scale for university students in Pakistani society drew this research study towards an exploration of the variable among Pakistani students. The purpose of the study is to comprehend the perceptions of university students about psychological flexibility. The experiences were collected in the form of written transcripts from 73 university students from two private universities in Lahore. Participants were recruited through purposive sampling. A qualitative summative content analysis approach was used to interpret the results of the data. A research question was formulated for data collection and codes were found using an operationalized definition from the literature review. The key findings that were highlighted through the content analysis were discussed based on the pre-determined codes in the operational definition of psychological
\end{abstract}

\footnotetext{
* PhD. Scholar, Clinical Psychology. School of Professional Psychology, University of Management and Technology. Senior Lecturer, Department of Radiological Sciences and Medical Imaging Technology (UIRSMIT), University College of Medicine and Dentistry (UCMD); Clinical Psychologist Placement Supervisor, Department of Psychology, LSPS, University of Lahore (UOL), Pakistan. Consultant Clinical Psychologist, Dr. Khalid Clinic, Gurumangat Road, Gulberg 3 Lahore. Email:nazish.idrees@rsmi.uol.edu.pk

† M.D. Psychiatry. Mayo Hospital, Lahore, Pakistan

₹ PhD. Hypnotherapy. Associate Professor, Department of Psychology, University of Lahore, Pakistan
} 
flexibility. After data analysis, the results provided four major themes. Themes derived through analysis are adaptive personality, assertive communication, emotion regulation and stress management. The results highlighted the concept of psychological flexibility in specific Pakistani population cultural context. The study provides direction to future researchers while working on the level of psychological flexibility in clinical and non-clinical practices.

Keywords: Psychological Flexibility; University Students; Content Analysis; Phenomenology; Credibility; Validity

\section{Introduction}

Psychological flexibility refers to an individual's ability to manage thoughts, emotions, and actions (Benoy et al., 2019). The change in this ability may predict the change in human behaviour. An existing model of change in behaviour that functions on the psychological flexibility model proposed as a part of acceptance and commitment therapy that is a form of traditional cognitive behavioural therapy (Hayes, 2019). The framework is similar to cognitive behavioural theory, through which irrational thoughts are identified and challenged with additional skills of acceptance, viewing the self as context, values-based committed action, and effective communication (Rodríguez Rey et al., 2019). The skills that are considered as a part of psychological flexibility explained in this model founded the effectiveness of a response to painful situations and experiences (McAteer \& Gillanders, 2019). It has been discovered that psychological flexibility may help in building resilience and coping with stress among adolescents and adults. A couple of scales have been developed based on this model of psychological flexibility validated for western countries (Benoy et al., 2019; Timmers, Simons, Hernandez, McCracken, \& Wallace, 2019). The scarcity of psychological flexibility assessment tools in an Asian Islamic has led to the planning for this study to find out the significant predictors of psychological flexibility in the university students of Pakistan (Meyer et al., 2019). Cultural differences are always present in subjective experiences due to contextual and social factors and they must take into consideration to understand the function of a concept within a specific population in a particular culture (Drake et al., 2019). It has been noticed that distress and experiential avoidance are manifested differently in different nations, to reach the unique expressions of psychological flexibility; exploration of the construct in specific context is crucial to assess and plan interventions for the enhancement of skills in managing stress among youth, improving their interpersonal relationships and communication for better performance. The literature review for the psychological flexibility leads to the understanding of the construct in terms of the - person by situation approach in which 
cognitive and emotional reappraisal demands the adaptability of human behavior based on a present situation. It refers to managing and regulating the expression of psychological functioning in human beings that reflects in the activities. Negative and unpleasant thoughts or emotions have been associated with psychosomatic symptoms, mood disruptions, anxiety, depression, and several other psychopathologies even psychological flexibility also has been shown that there is a potential to mediate the impact of psychedelic drugs on mental health and functioning. The trials containing the control group have been demonstrated that an increase in the tendency to manage own thoughts, emotions, and actions may exhibit different outcomes due to the influence of one's cultural, social, and environmental factors. This study helps in identifying the dimensions that are closely defining the role of psychological flexibility. This may lead to the planning of assessment procedures and training to improve relevant skills for the prevention of mental health issues among youth. The idea of psychological flexibility through the model of west is not applicable to explain psychological flexibility in eastern cultures due to differences in the values, social factors and other conditions.

\section{Purpose}

The purpose of the study was to illustrate the perceptions of private university students about psychological flexibility. This study is a part of indigenous scale development and validation for the university student population in Lahore, Pakistan. In phase I of the scale development process, phenomenology was explored for the given variable. Due to cultural and socio-demographical differences that are seen between Pakistan and other countries, there is a need to develop an indigenous scale and for that, it is crucial to extract the relevant information directly from the sample within the specific population.

\section{Method}

This study is qualitative research. To explore the perception related to psychological flexibility, phenomenology is the suitable approach because it directly related the expression with experiences of the target population (Neuendorf \& Jeffres, 2017; Skalski, Neuendorf, \& Cajigas, 2017). There are two types of phenomenology, transcendental (descriptive) and hermeneutic (interpretative). For this study hermeneutic phenomenology was considered due to the need to collect reflections from the target population based on their interpretation of the process of individuals with psychological flexibility in their life's world (Neubauer, Witkop, \& Varpio, 2019). Literature also recommended the selection of content analysis as a qualitative research method for the specific qualitative approach of 
phenomenology in the context of defining the function of a psychological construct to be explored.

To explore the phenomenon of psychological flexibility, content analysis was carried out. Content analysis is defined as the systematic and neutral analysis of the message's characteristics (Worley \& Krishnan, 2020). Many approaches come under qualitative content analysis (Kortegast, Jaekel, \& Nicolazzo, 2020). The summative approach is the most suitable content analysis form for this study in which the data analysis starts with the identification of frequently occurring words and content in the text collected for the study (Kindermann, 2020). This approach helps in understanding the contextual use of psychological flexibility. The main idea is to focus on the utility of the term "psychological flexibility" rather than interpreting the meaning or quantifying the codes as we do in other qualitative approaches (Karanikola, 2019) including interpretative phenomenological analysis and thematic analysis but this is a technique to gain inferences from the text that are replicable and valid. The textual material also known as manifest content is utilized for analysis as it appears in the written scripts. Latent content that counts as the hidden meaning in the text is noted and presented (Furlonger et al., 2019) in the form of a summary that deductively explains the alternative terms used to express the phenomenology of psychological flexibility. The advantages of this form of analysis include the researcher's bracketing, an unobtrusive and unbiased way to read and marked the scripts several times, to highlight relevant material that is common among the majority of the responses (Kindermann, 2020). The interest and insight about the phenomenon of the variable add attention and trustworthiness in the presentation of the data. Credibility and internal consistency of the data are revealed through consistent responses between the participants while the validation occurs with the help of an experienced professionals team (Haughton \& Singh, 2019).

The number of occurrences of similar meaning terms that were directly mentioned by the participants as verbatim was recorded. In this way, the homogeneity in the demographic characteristics of the participants maintains the clarity about the similar-looking terms (Meltzer \& Davy, 2019). The assumption is that the sample responses would be somewhat similar. Moreover, the credibility and accuracy of the codes and themes are finalized after the review of the scripts of the data (Serafini \& Reid, 2019). Then a group of experts validates the information based on their relevant experience to add more transferability to the given codes and themes with comparison across the participant's discussion. All the possible underlying meanings that were consistent throughout the scripts were taken in the initial list prepared for this study. The preferences of the results were then discussed in the light of comparisons with similar researches (Matt, Dietenberger, \& Weise, 2020). This form of analysis can be conducted without the use of any computer-based software. The final results were then explicitly showing the 
overlapping themes that emerged during the analysis were exhibited in the form of a table. All the patterns of recurring themes are contextualized on the pre-determined codes described by the purpose of exploration in the qualitative study (Haughton \& Singh, 2019).

A research question was formulated to explore the perceptions of university students about psychological flexibility based on an operational definition. Phenomenological content analysis is considered one of the big qualitative approaches. The theory behind this approach advised that questions used to explore the perceptions interact with a social process (Korstjens \& Moser, 2017). The operational definition was derived through a recent literature review as follows: the ability to attempt a change in one's thoughts, emotions, and actions. The research question was "in your view, what are the characteristics of the people who can adjust their thoughts, feelings, and actions based on the present situation?". In this way, the interview influences the research process (Hsieh \& Shannon, 2005).

The application of this particular methodology focused on the appropriate sampling technique. The non-probability sampling strategy was carried out to determine the sample because the qualitative sample is smaller and randomization is not feasible to do. Additionally, an article reviewed various studies and pointed out those two kinds of non-random sampling, convenience and purposive are good to follow based on the nature and kind of research. It was decided then that the purposive sampling would be better based on the nature of this study to extract views related to experiences with psychologically flexible individuals (Etikan, Musa, \& Alkassim, 2016).

A sample of 73 students, both males and females between 18-22 years old who were enrolled in a Bachelor's degree from various disciplines of natural, social, and management sciences responded to the research questions. Previous literature proposed that the sample range from minimum to maximum anywhere based on availability and the quality of responses. Minimum 5 participants are suggested but the responses initially gathered were one-worded or phrases only with several unique explanations that led the research to expand it more till saturation occurs (Boddy, 2016). Verbal informed consent was sought that ensured them their confidentiality. To maintain the privacy of the participants and data, no personal information was recorded that may identify the participants of this study. The verbatim was noted and the written scripts were then utilized for content analysis. The researcher's bracket played a role in taking out the relevant interpretations that defines the characteristics of a psychologically flexible individual. This procedure was carefully planned for this study mainly because to eliminate foreseen biases guided by previous research studies (Mehra, 2002).

The summative coding approach was used for data analysis in this study. After a theoretical background and framework rationale justified the use of this technique for data analysis of this study, the conceptualization of the variable along with an operational definition was given (Hsieh \& 
Shannon, 2005). Sampling was done that follows data collection and credibility check and validation during the analysis (Pyett, 2003). The procedure of emerging themes started with extracting the keywords and phrases from the responses to the research question from the participants of the study. The main codes for this study were identified both before and during the data analysis. Codes for this study were attained through the definition of psychological flexibility. The interest of the researcher in this study was to extract the common thoughts, emotions, and actions that people with psychological flexibility might experience. Finally, the common themes and sub-theme categories were mentioned (Berlin, Rosander, Frykedal, Törnkvist, \& Barimani, 2020). The results are discussed in detail with examples from the text in the participants' scripts.

The view and experiences of the participants about psychological flexibility served as the important contributors to the development of the scale to measure the level of psychological flexibility in university students. Furthermore, a group of five experts in student counselling, clinical psychologists, and mental health experts was selected with 2 years of relevant experience and they were requested to rate the derived themes and sub-theme categories to strengthen the outcome (Chenail, 2011). The majority of the experts showed an agreement of $90 \%$ and above with the responses by the participants (Pyett, 2003). The initial list of the phrases was more than 465 in number that was then skimmed down to 64 phrases and finally a list of 43 comprehensive characteristics of psychological flexibility was attained. The content analysis of the data provided four major themes for psychological flexibility that covers and related to the numerous other sub-theme categories from the available text.

The following steps (given in Fig. 1) were followed to complete the content analysis of the data.

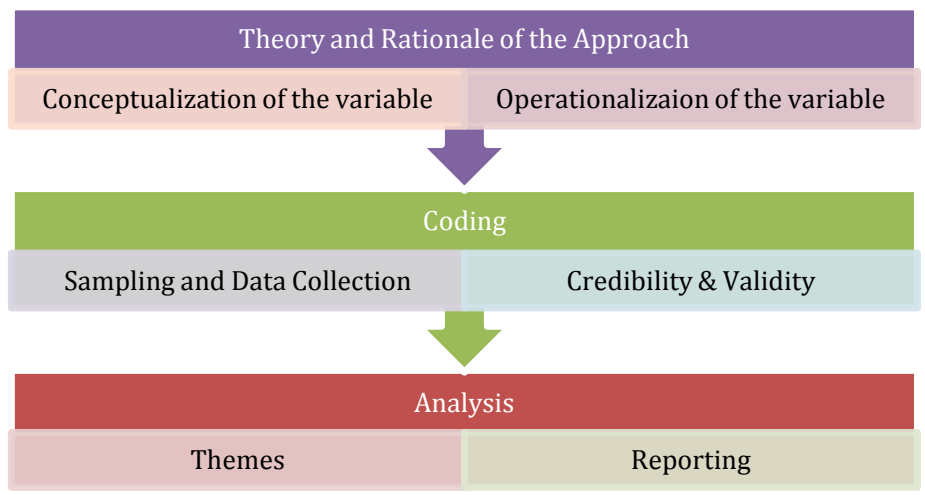

Figure 1: Steps for Content Analysis adopted from a work by Neuendorf, K. A. (2017)

\section{Results}


Three codes were determined based on the concept and operational definition of psychological flexibility before the data analysis with the use of content analysis. The four themes that emerged from the text collected by the participants of the study, are: (a) adaptive personality, (b) stress management, (c) emotion regulation, and (d) assertive communication. These themes were comprised of the set of similar meaning bundles of keywords and phrases that comes under the four main categories with occurrence in more than $50 \%$ of the responses. The majority of the respondents agree with the presence of these characteristics below (in Table 1.).

Table 1: A Summary of the Data Analysis for this Study using a Summative Approach to Content Analysis- Psychological Flexibility Phenomenology

\begin{tabular}{|c|c|c|}
\hline Codes & Major Themes & Sub-Categories \\
\hline \multirow{5}{*}{ Thoughts } & Adaptive personality & Good decision-maker (91\%) \\
& & Focused on the present (90\%) \\
& & Admit mistakes (90\%) \\
& & Grateful (78\%) \\
& & Strong faith (58\%) \\
& Stress management & Believe in self (93\%) \\
& & Listen actively (88\%) \\
& & Prepared to face challenges (59\%) \\
& & Accept change (55\%) \\
& & Offer prayers (67\%) \\
& & Forgiving (76\%) \\
& & Understand others (87\%) \\
& Emotion regulation & Control anger (93\%) \\
& & Tolerance (95\%) \\
& & Respectful (96\%) \\
& & Smiling face (79\%) \\
& & Effective dealing with others (84\%) \\
& & Helpful (89\%) \\
& & Trustworthy (66\%) \\
& & Honest (72\%) \\
& & Kind (73\%) \\
& & Strong (85\%) \\
& & Avoid conflicts (77\%) \\
& & Good relationships $(60 \%)$ \\
& & Productive social interaction (91\%) \\
\hline
\end{tabular}

\section{Adaptive Personality}

The examples of the perceptions under this theme were expressed as follows: they "focus on achievements", "have a clear vision", "and learn from bad experiences in past and work for future", "they are creative", "they are focused on their goals", "these people can adjust in every environment", "they 
can make good decisions by analyzing the situation", "they are truthful to others", "they are attentive in situations", "they actively help others when anyone needs", take responsibility of own actions" and "they listen carefully".

The following narratives were coherent with the themes and the verbatim given to that describes adaptive personality (Rahimi, Meratian, \& Mahmoodabadi, 2018). The participants shared that psychological flexibility is naturally present in some individuals. In participant's view, the adaptive personality focus on present moments (Kercood, Lineweaver, Frank, \& Fromm, 2017), analyze their thoughts and make decisions appropriately based on what the present situation demands. Admitting mistakes is another response that frequently associated with adaptive personality (Puolakanaho, Tolvanen, Kinnunen, \& Lappalainen, 2020) in the results of phenomenology. Other closely related categories that come under this theme are attentive listening, helping, showing trustworthiness, and honesty for others (Fincham, Rogge, \& Beach, 2018).

\section{Stress Management}

The example of the perceptions under this theme was expressed as follows: they "accept the challenges of life", "appreciate each moment", "they are self-directed", "they give attention", "they mould their thoughts", they are independent and open-minded", "they find solutions", "they are emotionally stable", "they desire for stimulating and exciting life", "they tolerate other people's behaviour", "they are mentally prepared to face challenges in future" and " they accept daily changes".

The following narratives were coherent with the themes and the verbatim given to that describes stress management (Asikainen, 2018). The participants shared that psychologically flexible individuals smile often, express positive emotions of joy and happiness that allows them to manage stress and control their anger. Tolerance is also one of the responses that frequently used by the participants. Other closely related categories that come under this theme are accepting of change and preparedness to face challenges (Singh \& O'Brien, 2020).

\section{Emotion Regulation}

The perceptions under this theme were expressed as follows: they are "positive", "aware about self", "observe emotions", "they value safety and stability", "they have complete control over their emotions and their reaction is their strength", "they stay calm in every situation", "they handle their bad temper" and "they offer prayers daily with strong faith".

The following narratives were coherent with the themes and the verbatim given to that describes emotion regulation. The participants described that psychological flexibility includes the presence of kindness, 
satisfaction (Fincham et al., 2018), gratefulness, and forgiveness in individuals. This indicates that the presence of the given moral attributes may help an individual in staying relaxed even when circumstances in life change (Frinking et al., 2019). Other closely related categories that come under this theme are feeling confident, having strong faith, and humbleness.

\section{Assertive Communication}

The examples under this theme were expressed as follows: they have "good communication skills", "social skills", "show cooperation", "respectful to others", "they are very polite", "they can influence others", "they don't want to create any issue", "they are courteous", "motivate others", "they know how to speak with aggressive people" and "they care about others".

The following narratives were coherent with the themes and the verbatim given to that describes assertive communication. Participants stated that in every situation, psychological flexibility promotes understanding of other people and prevents arguments in relationships (Benjamin, HarbeckWeber, Ale, \& Sim, 2020). According to the participant's experience, showing respect to others while they have a different point of view is possible with the presence of psychological flexibility. Other closely related categories that come under this theme are effective in dealing with people, avoidance of conflicts, and maintaining stable relationships with others (Jansen et al., 2017). Alternative responses were collated together, overlapped ideas were skipped and the most commonly occurring characteristics were labelled as a part of one of the four dominant themes.

\section{Discussion}

Every theme was associated with each of the codes derived from the literature review. The university students viewed the characteristics of psychological flexibility as one of the following: thought, emotion, or action. The verbatim obtained through the responses of the participants were labelled in a way that relates to being an adaptive personality, able to manage stress easily, able to control emotions and emotional reactions or urges and with the assertive communication in which an individual might express own idea to others in the most respectful way without compromising the right of self or others. The output following the review of experts for validation added even more trustworthiness to the themes and subcategories. The items generated as a result of the analyses comprises of statements for the scale, for example; to keep eyes on capabilities, to act differently for change in outcomes, to show cooperation with others when needed, to observe emotions, to feel grateful, to motivate others, to respect others point of view and so on.

It has been reviewed through recent literature on the development and 
validation of psychological flexibility inventory (Rolffs, Rogge, \& Wilson, 2018) for students in European countries that psychological flexibility is multi-dimensional (Scott, McCracken, \& Norton, 2016; Seidler, Stone, Clark, Koran, \& Drake, 2020). This is consistent with the codes of our study that covered the thoughts, feelings, and actions of a psychologically flexible individual. Furthermore, the themes and sub-categories of this study were all potentially relevant to either of the codes. The structure of psychological flexibility in Pakistani culture and the university population was aligned with other populations and consistent in other cultures.

The psychological flexibility model supports the ability to accept and change in the process of daily living, this idea assisted the adaptive personality theme that emerged in this study's data analysis (Watts \& Luoma, 2019). A cultural exploration among Native Americans for psychological flexibility has produced evidence of linking psychological flexibility for healing through a potentially altered state of consciousness as seen in the responses that showed attention, focus, creativity, and decision making (Sabucedo, 2017). This illustrated that temporary changes in the state of mind expressed adaptive personality hence confirming the trustworthiness of the results.

The studies that have verified the effective clinical outcome of psychological flexibility in patients suffering from chronic pain (Bakhurst, Loew, McGuire, Halford, \& Markman, 2017) with the use of acceptance of the present situation and handling pain with control over emotions (Abbasi, 2017) that are consistent with the stress management theme of psychological flexibility phenomenology that was derived through the content analysis in this study (Scott, Hann, \& McCracken, 2016; Yang, McCracken, \& MossMorris, 2017). It plays a great role as a dimension of acceptance and commitment therapeutic approach, stress and well-being have been linked to psychological flexibility in several studies (Wersebe, Lieb, Meyer, Hofer, \& Gloster, 2018).

Similarly, grief and other psychological symptoms i.e. depression is mediated by psychological flexibility and it also moderated between traumatic symptoms and daily pain (Berghoff, McDermott, \& DixonGordon, 2018) by as elaborated in the emotion regulation theme that explains the presence of positive emotions as psychological flexibility (BaŞ, Selva, YÜKsel, \& Güngör, 2019; Davis, Barrett, \& Griffiths, 2020). The motivation might increase (Mikaeli, Rahimi, \& Sedaghat, 2018) when individuals experience psychological flexibility, this is again highlighting that emotion regulation (Harrison, 2019) theme responses and categories are related to the phenomenon strongly (McCracken \& Scott, 2018).

Psychosocial outcomes in children at developmental stages were studied under psychological flexibility (Van Schoors et al., 2019), the theme of assertive communication and the responses related to communication and social skills (Faal, Vahid, Taime, Shaban, \& Amani, 2019) were found to be 
relevant as well (Brassell et al., 2016).

In other words, it has been predicted through the consistent literature (Ramaci, Bellini, Presti, \& Santisi, 2019; Rosado, 2017) evidence for the emerged themes in the study that psychological flexibility is a protective factor for negative emotions (Marshall \& Brockman, 2016), unstable interpersonal relationships (Boatemaa, Oppong Asante, \& Agyemang, 2019) and related issues (Godfrey et al., 2019). The dependability of the results in this study is strengthening through the studies (Foote, Hamer, Roland, Landy, \& Smitherman, 2016) relevant to the phenomenon of psychological flexibility in a wide range of perspectives (Almarzooqi, Chilcot, \& McCracken, 2017; Lin, Klatt, McCracken, \& Baumeister, 2018).

\section{Ethics}

The following ethics were followed and maintained for this study, informed consent (Höglund \& Falkenström, 2018), confidentiality, and anonymity as instructed in the latest codes of conduct guidelines from the American Psychological Association (Young, 2017).

\section{Limitations and Recommendations}

The data responses came from students of the private sector who were undergraduates. Although the sample represents the university population very well because the students belong to diverse backgrounds, the role of a specific culture is seen in the majority of the responses. A high level of saturation achieved earlier than expected marked a large number of responses. It is recommended that phenomenology can be analyzed in the future for the same variable using different qualitative approaches. For this study, phenomenology was analyzed as a small phase of psychological flexibility scale development for a specific population in the Pakistani culture.

\section{Conclusion}

The qualitative content analysis in this study was aimed at the development and validation of indigenous scale development. However, it opened the path for future researchers to consider further descriptive work on the same variable in students from different demographics. The study was ethically sound and credible due to the measures that were taken into account since the beginning of the project.

The outcome of the content analysis using a summative approach and expert's validation review allowed obtaining a phenomenon of psychological flexibility in Pakistani university students. The results of the study had been thoroughly consistent with the concepts of psychological flexibility worldwide to some extent in the available literature about theory and conceptual 
framework of psychological flexibility. The conceptual clarity of the construct was achieved to a great extent. This will be useful for further work on psychological flexibility based clinical and non-clinical interventions and utility nationwide for a variety of health problems and illnesses.

\section{Implications of the Study}

This study suggested few practical implications in the clinical and nonclinical fields. The data integrated reliable and valid information about the beliefs and observations of the university population relevant to psychological flexibility. This implies the need for further research to develop culturally appropriate measuring tools for the assessment of this ability. For future researchers, following the assessment of psychological flexibility skill-based training must be created to improve social adjustment, moral values, compassion and self-care among youth. The results have highlighted the utilization of psychological flexibility in education and clinical settings for the therapists and teaching staff in helping the individuals to learn to manage themselves during crises and enhance effective communication in interpersonal relationships. Finding out the prevalence of psychological flexibility in the Pakistani population and its relation with educational degrees and professions is recommended. Furthermore, health professionals can focus on psychological flexibility studies using quantitative approaches, experimental designs and sample from a variety of contexts and diverse populations.

\section{Ethical Review Statement}

This study has been passed by the institutional review committees of the respective institution and the national regulating bodies. A permission letter has been signed by the higher authorities to recruit participants from the university.

\section{Disclaimer}

This study has not been presented or published anywhere else.

\section{Conflict of Interest}

None to declare.

\section{Funding}

None to declare. 


\section{References}

Abbasi, I.S. (2017). Personality and marital relationships: Developing a satisfactory relationship with an imperfect partner. Contemporary Family Therapy, 39(3), 184-194.

Almarzooqi, S., Chilcot, J., \& McCracken, L.M. (2017). The role of psychological flexibility in migraine headache impact and depression. Journal of Contextual Behavioral Science, 6(2), 239-243.

Asikainen, H. (2018). Examining indicators for effective studying-the interplay between student integration, psychological flexibility and selfregulation in learning. Psychology, Society, \& Education, 10(2), 225237.

Bakhurst, M.G., Loew, B., McGuire, A.C.L., Halford, W.K., \& Markman, H.J. (2017). Relationship education for military couples: Recommendations for best practice. Family process, 56(2), 302-316.

BaŞ, S., Selva, Ü., YÜKsel, Ö., \& Güngör, D. (2019). Mediator role of psychological flexibility in the relationship between traumatic grief and psychological symptoms. Depression, 19, 10.

Benjamin, J.Z., Harbeck-Weber, C., Ale, C., \& Sim, L. (2020). Becoming flexible: Increase in parent psychological flexibility uniquely predicts better well-being following participation in a pediatric interdisciplinary pain rehabilitation program. Journal of Contextual Behavioral Science.

Benoy, C., Knitter, B., Knellwolf, L., Doering, S., Klotsche, J., \& Gloster, A.T. (2019). Assessing psychological flexibility: Validation of the open and engaged state questionnaire. Journal of Contextual Behavioral Science, 12, 253-260.

Berghoff, C.R., McDermott, M.J., \& Dixon-Gordon, K.L. (2018). Psychological flexibility moderates the relation between ptsd symptoms and daily pain interference. Personality and Individual Differences, 124, 130-134.

Berlin, A., Rosander, M., Frykedal, K.F., Törnkvist, L., \& Barimani, M. (2020). Fatherhood group sessions: A descriptive and summative qualitative study. Nursing \& Health Sciences, 22(4), 1094-1102.

Boatemaa, M.A., Oppong Asante, K., \& Agyemang, C.B. (2019). The moderating role of psychological flexibility in the relationship between organizational commitment, workaholism, job security, and corporate entrepreneurship among information technology workers in accra, ghana. SAGE Open, 9(3), 2158244019871063.

Boddy, C.R. (2016). Sample size for qualitative research. Qualitative Market Research: An International Journal.

Brassell, A.A., Rosenberg, E., Parent, J., Rough, J.N., Fondacaro, K., \& Seehuus, M. (2016). Parent's psychological flexibility: Associations with parenting and child psychosocial well-being. Journal of Contextual Behavioral Science, 5(2), 111-120. 
Chenail, R.J. (2011). Interviewing the investigator: Strategies for addressing instrumentation and researcher bias concerns in qualitative research. Qualitative Report, 16(1), 255-262.

Davis, A.K., Barrett, F.S., \& Griffiths, R.R. (2020). Psychological flexibility mediates the relations between acute psychedelic effects and subjective decreases in depression and anxiety. Journal of Contextual Behavioral Science, 15, 39-45.

Drake, C.E., Masuda, A., Dalsky, D., Stevens, K.T., Kramer, S., Primeaux, S.J., . . . Mitamura, T. (2019). Examining us and japanese college students' differences in psychological distress: The mediating roles of valued action and experiential avoidance. International Journal for the Advancement of Counselling, 41(1), 25-40.

Etikan, I., Musa, S.A., \& Alkassim, R.S. (2016). Comparison of convenience sampling and purposive sampling. American journal of theoretical and applied statistics, 5(1), 1-4.

Faal, M., Vahid, T.A., Taime, Z.M., Shaban, N., \& Amani, O. (2019). The effectiveness of accepted and commitment group therapy on psychological flexibility and family communication patterns in women with type 2 diabetes. Iranian Journal of Psychiatric Nursing, 7(4), 4149.

Fincham, F.D., Rogge, R., \& Beach, S.R.H. (2018). Relationship satisfaction.

Foote, H.W., Hamer, J.D., Roland, M.M., Landy, S.R., \& Smitherman, T.A. (2016). Psychological flexibility in migraine: A study of pain acceptance and values-based action. Cephalalgia, 36(4), 317-324.

Frinking, E., Jans-Beken, L., Janssens, M., Peeters, S., Lataster, J., Jacobs, N., \& Reijnders, J. (2019). Gratitude and loneliness in adults over 40 years: Examining the role of psychological flexibility and engaged living. Aging \& mental health, 1-8.

Furlonger, B., Ostojic, M., Chung, J., Philips, K., Busacca, M., Moore, D., \& Anderson, A. (2019). A framework to assist school psychologists and counsellors in recommending quality apps for supporting diabetes selfmanagement: An illustrative assessment using content analysis. Journal of Psychologists and Counsellors in Schools, 29(1), 82-97.

Godfrey, K.M., Butryn, M.L., Forman, E.M., Martinez, M., Roberts, S.R., \& Sherwood, N.E. (2019). Depressive symptoms, psychological flexibility, and binge eating in individuals seeking behavioral weight loss treatment. Journal of Contextual Behavioral Science, 14, 50-54.

Harrison, A.M. (2019). Exploring longitudinal relationships between psychological flexibility and medication adherence, mood and general functioning in people with long-term health conditions. University of Leeds.

Haughton, N.A., \& Singh, P.R. (2019). A content analysis of the emergent scholarship on digital and open badges in higher education learning and 
assessment. Issues and Trends in Learning Technologies, 7(2).

Hayes, S.C. (2019). Acceptance and commitment therapy: Towards a unified model of behavior change. World psychiatry, 18(2), 226.

Höglund, A.T., \& Falkenström, E. (2018). The status of ethics in swedish health care management: A qualitative study. BMC health services research, 18(1), 608.

Hsieh, H.-F., \& Shannon, S.E. (2005). Three approaches to qualitative content analysis. Qualitative health research, 15(9), 1277-1288.

Jansen, J.E., Haahr, U.H., Lyse, H.-G., Pedersen, M.B., Trauelsen, A.M., \& Simonsen, E. (2017). Psychological flexibility as a buffer against caregiver distress in families with psychosis. Frontiers in psychology, 8 , 1625.

Karanikola, M.N.K. (2019). Content analysis in critical and emergency care: A discussion paper. CONNECT: The World of Critical Care Nursing, 13(1).

Kercood, S., Lineweaver, T.T., Frank, C.C., \& Fromm, E.D. (2017). Cognitive flexibility and its relationship to academic achievement and career choice of college students with and without attention deficit hyperactivity disorder. Journal of Postsecondary Education and Disability, 30(4), 329-344.

Kindermann, K. (2020). Summative content analysis as a core method to reconstruct subjective theories using structure-formation-techniques. Paper presented at the Forum Qualitative Sozialforschung/Forum: Qualitative Social Research.

Korstjens, I., \& Moser, A. (2017). Series: Practical guidance to qualitative research. Part 2: Context, research questions and designs. European Journal of General Practice, 23(1), 274-279.

Kortegast, C.A., Jaekel, K.S., \& Nicolazzo, Z. (2020). Thirty years of lgbtq pre-publication knowledge production in higher education research: A critical summative content analysis of ashe conference sessions. Journal of Homosexuality, 1-25.

Lin, J., Klatt, L.-I., McCracken, L.M., \& Baumeister, H. (2018). Psychological flexibility mediates the effect of an online-based acceptance and commitment therapy for chronic pain: An investigation of change processes. Pain, 159(4), 663-672.

Marshall, E.-J., \& Brockman, R.N. (2016). The relationships between psychological flexibility, self-compassion, and emotional well-being. Journal of Cognitive Psychotherapy, 30(1), 60-72.

Matt, F.J., Dietenberger, M.A., \& Weise, D.R. (2020). Summative and ultimate analysis of live leaves from southern us forest plants for use in fire modeling. Energy \& Fuels.

McAteer, G., \& Gillanders, D. (2019). Investigating the role of psychological flexibility, masculine self-esteem and stoicism as predictors of psychological distress and quality of life in men living with 
prostate cancer. European journal of cancer care, 28(4), e13097.

McCracken, L.M., \& Scott, W. (2018). Motivation from the perspective of contextual cognitive behavioral approaches and the psychological flexibility model.

Mehra, B. (2002). Bias in qualitative research: Voices from an online classroom. The qualitative report, 7(1), 1-19.

Meltzer, A., \& Davy, L. (2019). Opportunities to enhance relational wellbeing through the national disability insurance scheme: Implications from research on relationships and a content analysis of ndis documentation. Australian Journal of Public Administration, 78(2), 250-264.

Meyer, E.C., Kotte, A., Kimbrel, N.A., DeBeer, B.B., Elliott, T.R., Gulliver, S.B., \& Morissette, S.B. (2019). Predictors of lower-than-expected posttraumatic symptom severity in war veterans: The influence of personality, self-reported trait resilience, and psychological flexibility. Behaviour research and therapy, 113, 1-8.

Mikaeli, N., Rahimi, S., \& Sedaghat, M. (2018). The role of family communication patterns, motivational structure and psychological flexibility in predicting couples' emotional divorce. Family Pathology, Counseling and Enrichment Journal, 4(1), 1-16.

Neubauer, B.E., Witkop, C.T., \& Varpio, L. (2019). How phenomenology can help us learn from the experiences of others. Perspectives on medical education, 8(2), 90-97.

Neuendorf, K.A., \& Jeffres, L.W. (2017). Media effects: Accounts, nature, and history of. The International Encyclopedia of Media Effects, 1-13.

Puolakanaho, A., Tolvanen, A., Kinnunen, S.M., \& Lappalainen, R. (2020). A psychological flexibility-based intervention for burnout: A randomized controlled trial. Journal of Contextual Behavioral Science, $15,52-67$.

Pyett, P.M. (2003). Validation of qualitative research in the "real world". Qualitative health research, 13(8), 1170-1179.

Rahimi, M., Meratian, N., \& Mahmoodabadi, H.Z. (2018). The role of family communication dimensions in adolescents' depression with the mediation of cognitive flexibility. Journal of Fundamentals of Mental Health, 20(5).

Ramaci, T., Bellini, D., Presti, G., \& Santisi, G. (2019). Psychological flexibility and mindfulness as predictors of individual outcomes in hospital health workers. Frontiers in psychology, 10, 1302.

Rodríguez Rey, R., Montesinos, F., Páez, M., McCracken, L., Núñez, S., González, C., . . . Hernando, A. (2019). Communication skills in the context of psychological flexibility: Training is associated with changes in responses to chronic pain in physiotherapy students in spain.

Rolffs, J.L., Rogge, R.D., \& Wilson, K.G. (2018). Disentangling components of flexibility via the hexaflex model: Development and 
validation of the multidimensional psychological flexibility inventory (mpfi). Assessment, 25(4), 458-482.

Rosado, M.A. (2017). Evaluating the relationship between psychological flexibility, teacher self-efficacy, teacher-student interactions, and burnout syndrome: Southern Illinois University at Carbondale.

Sabucedo, P. (2017). The psychological flexibility model from a cultural perspective: An interpretative analysis of two native american healing rituals. International Journal of Culture and Mental Health, 10(4), 367375.

Scott, W., Hann, K.E.J., \& McCracken, L.M. (2016). A comprehensive examination of changes in psychological flexibility following acceptance and commitment therapy for chronic pain. Journal of contemporary psychotherapy, 46(3), 139-148.

Scott, W., McCracken, L.M., \& Norton, S. (2016). A confirmatory factor analysis of facets of psychological flexibility in a sample of people seeking treatment for chronic pain. Annals of Behavioral Medicine, 50(2), 285-296.

Seidler, D., Stone, B., Clark, B.E., Koran, J., \& Drake, C.E. (2020). Evaluating the factor structure of the multidimensional psychological flexibility inventory: An independent replication and extension. Journal of Contextual Behavioral Science.

Serafini, F., \& Reid, S.F. (2019). Multimodal content analysis: Expanding analytical approaches to content analysis. Visual Communication, 1470357219864133.

Singh, R.S., \& O'Brien, W.H. (2020). The impact of work stress on sexual minority employees: Could psychological flexibility be a helpful solution? Stress and Health, 36(1), 59-74.

Skalski, P.D., Neuendorf, K.A., \& Cajigas, J.A. (2017). Content analysis in the interactive media age. The content analysis guidebook, 2, 201-242.

Timmers, I., Simons, L.E., Hernandez, J.M., McCracken, L.M., \& Wallace, D.P. (2019). Parent psychological flexibility in the context of pediatric pain: Brief assessment and associations with parent behaviour and child functioning. European Journal of Pain, 23(7), 1340-1350.

Van Schoors, M., De Paepe, A.L., Lemiere, J., Morez, A., Norga, K., Lambrecht, K., . . . Verhofstadt, L.L. (2019). Family adjustment when facing pediatric cancer: The role of parental psychological flexibility, dyadic coping and network support. Frontiers in Psychology, 10, 2740.

Watts, R., \& Luoma, J. (2019). The use of the psychological flexibility model to support psychedelic assisted therapy. Journal of Contextual Behavioral Science.

Wersebe, H., Lieb, R., Meyer, A.H., Hofer, P., \& Gloster, A.T. (2018). The link between stress, well-being, and psychological flexibility during an acceptance and commitment therapy self-help intervention. International Journal of Clinical and Health Psychology, 18(1), 60-68. 
Worley, J., \& Krishnan, D. (2020). Fighting the battle of recovery together: A content analysis of anonymous posts in an online substance use forum. Issues in Mental Health Nursing, 41(2), 102-112.

Yang, S.-Y., McCracken, L.M., \& Moss-Morris, R. (2017). Psychological treatment needs for chronic pain in singapore and the relevance of the psychological flexibility model. Pain Medicine, 18(9), 1679-1694.

Young, G. (2017). Revising the apa ethics code: Springer. 